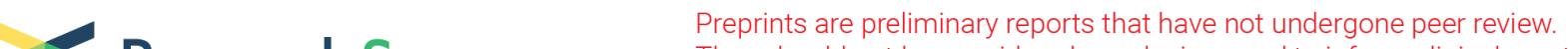 Research Square They should not be considered conclusive, used to inform clinical practice, or referenced by the media as validated information.
}

\section{The reciprocal loop of MALAT1 and HIF-1a facilitates gemcitabine resistance in pancreatic cancer by enhancing glycolysis}

\section{Zhengle Zhang}

Renmin Hospital of Wuhan University: Wuhan University Renmin Hospital

Han Han

Central Hospital of Wuhan

\section{Qiong Gong}

Renmin Hospital of Wuhan University: Wuhan University Renmin Hospital

\section{Yuping Rong}

Renmin Hospital of Wuhan University: Wuhan University Renmin Hospital

\section{Hanjun Li}

Renmin Hospital of Wuhan University: Wuhan University Renmin Hospital

\section{Zhongchao Zhu}

Renmin Hospital of Wuhan University: Wuhan University Renmin Hospital

\section{Pei Mei}

Renmin Hospital of Wuhan University: Wuhan University Renmin Hospital

\section{Chuanbing Zhao}

Renmin Hospital of Wuhan University: Wuhan University Renmin Hospital

\section{Zhigang Tang}

Renmin Hospital of Wuhan University: Wuhan University Renmin Hospital Jing Tao ( $\nabla$ tjwdrm@163.com )

Renmin Hospital of Wuhan University https://orcid.org/0000-0002-8947-7520

\section{Research}

Keywords: Gemcitabine, Glycolysis, MALAT1, HIF-1a, Pancreatic cancer

Posted Date: December 29th, 2020

DOI: https://doi.org/10.21203/rs.3.rs-135235/v1

License: (1) (1) This work is licensed under a Creative Commons Attribution 4.0 International License. Read Full License 


\section{Abstract \\ Background}

Profound chemoresistance is a prominent and intractable problem in pancreatic cancer. Gemcitabine, a first-line chemotherapeutic drug for pancreatic cancer, has provided only minimal benefits for patients; however, the underlying mechanisms remain to be investigated. Enhanced aerobic glycolysis has been suggested to be correlated with the phenotype of drug resistance, while MALAT1 has been indicated to be associated with poor gemcitabine response. However, the implicated regulatory mechanisms still need to be further explored.

\section{Methods}

The mRNA level of MALAT1, HIF-1a, and glycolytic enzymes were analyzed by qRT-PCR. The protein level of HIF-1 $a$ and glycolytic enzymes were detected by western blot analysis. The glucose uptake and lactate level were measured by glucose uptake and lactate production assay, respectively. The cell apoptosis was evaluated by apoptosis analysis using Annexin V/propidium iodide ( $\mathrm{PI}$ ) staining. The sensitivity to gemcitabine was measured by MTT assay. The binding of HIF-1a to the promoter of MALAT1 was tested by ChIP assay. The xenograft model of pancreatic cancer was established to examine the combined effect of MALAT1 knockdown and gemcitabine chemotherapy in vivo.

\section{Results}

In this study, we showed that low concentrations of gemcitabine promoted the expression of key glycolytic enzymes, such as GLUT1, HK2, and LDHA, as well as glucose uptake and lactate production in pancreatic cancer cells, while inhibition of glycolysis suppressed gemcitabine chemoresistance. In addition, we found that MALAT1 expression was induced by gemcitabine and mediated enhanced glycolysis and chemoresistance. Silencing MALAT1 inhibited the level of gemcitabine-induced HIF-1a expression and reinforced gemcitabine-induced apoptosis in vitro and in vivo. Furthermore, HIF-1 a could bind the promoter of MALAT1 transcriptionally and mediate the glycolysis induced by gemcitabine.

\section{Conclusions}

Our data suggest a critical role of glycolytic metabolism in acquired gemcitabine resistance through the positive feedback loop of MALAT1 and the HIF-1 a signaling pathway. Our results will provide a basis for developing potential therapeutic targets to reverse gemcitabine resistance.

\section{Background}


Pancreatic cancer is one of the most devastating digestive tumors worldwide, with an almost equal incidence and mortality rate [1]. Despite strenuous endeavors, the overall prognosis has not been improved much in comparison with that of other malignancies. The majority of patients with pancreatic cancer are diagnosed at an inoperable disease stage due to obscure symptoms. Therefore, the options available for patients with pancreatic cancer are quite few except for chemotherapy. However, unfortunately, chemoresistance is an intractable clinical challenge. For example, gemcitabine, the first-line recommended drug, exhibits limited efficacy in treating pancreatic cancer. Thus, illuminating the mechanisms involved in resistance will have great significance in combating pancreatic cancer.

It has been noted for decades that tumor cells can rapidly utilize glucose to convert lactate and adenosine triphosphate (ATP) even in the presence of a normal oxygen supply, which is characterized as aerobic glycolysis or the "Warburg effect" [2]. Glycolytic flux not only provides cancer cells with energy but also produces biomass for metabolic biosynthesis, thus meeting the demands of aggressive proliferation. In addition, the intermediates from the branches of glycolysis contribute to cellular redox homeostasis [3]. Accumulating evidence indicates that aerobic glycolysis confers cancer cells with a phenotype of drug resistance [4], and targeting glycolysis can restore the sensitivity of pancreatic cancer to gemcitabine [5]. On the other hand, a recent study reported that gemcitabine treatment may promote the metabolic reprogramming of pancreatic cancer cells toward aerobic glycolysis [6]. However, the explicit underlying mechanism still needs to be further investigated. Elucidation of the regulatory mechanism of aerobic glycolysis will facilitate enhanced chemosensitivity to gemcitabine in patients with pancreatic cancer.

Long noncoding RNAs (IncRNAs) are a series of transcripts longer than 200 nt with no protein-coding capacity. Initially recognized as "transcriptional noise", IncRNAs have been shown to participate in the initiation, development, and progression of cancers in a variety of ways [7]. In addition, aberrantly expressed IncRNAs have been closely implicated in chemoresistance and radioresistance [8, 9]. For example, IncRNA SLC7A11-AS1 was reported to be overexpressed and promote gemcitabine resistance by scavenging ROS in pancreatic cancer [10]. Intriguingly, gemcitabine treatment could induce the IncRNA HOTAIR to enhance the drug resistance and stemness of pancreatic cancer [11]. Thus, it might be a reasonable approach to explore these IncRNAs induced by gemcitabine and the involved mechanisms to find new targets that can reverse gemcitabine resistance.

Metastasis-associated lung adenocarcinoma transcript 1 (MALAT1) is a highly evolutionarily conserved IncRNA [12] that was initially identified as overexpressed in metastatic non-small-cell lung cancer tissues [13]. Recent studies have shown that MALAT1 is aberrantly expressed in numerous cancers and can function as an oncogene or tumor suppressor gene depending on the cancer type $[14,15]$. In pancreatic cancer, MALAT1 was verified to be overexpressed and promote the growth, migration, invasion, and stemness of cancer cells [16-18]. In addition, a high level of MALAT1 has been indicated to be inversely correlated with gemcitabine response and progression-free survival (PFS) [19]. However, the relationship between gemcitabine treatment and MALAT1 is not fully understood. Our preliminary study found that a low concentration of gemcitabine promoted the level of MALAT1 in pancreatic cancer cells in a dose- 
dependent manner. We therefore speculated that the induction of MALAT1 might be an important mechanism of acquired gemcitabine chemoresistance. MALAT1 has been shown to enhance glycolysis and inhibit gluconeogenesis in hepatocellular carcinoma via TCF7L2 [20]. Hypoxia inducible factor 1a (HIF-1a) is considered a key factor in mediating glycolysis, while MALAT1 could stabilize HIF-1a and enhance arsenite-induced glycolysis in human hepatic cells [21]. On the other hand, MALAT1 could be upregulated through the hypoxia-induced HIF-1a signaling pathway in endometrial stromal cells [22], suggesting that there might be a reciprocal regulation between MALAT1 and HIF-1a.

In the present study, we verified that gemcitabine treatment reinforced acquired chemoresistance by enhancing glycolic flux through a positive feedback loop comprising MALAT1 and HIF-1a. Our results provide new insights into the resistance mechanisms and potential targets for restoring gemcitabine sensitivity.

\section{Methods}

\section{Cell culture}

The two human pancreatic cancer cell lines SW1990 and PANC-1 used in this study were obtained from the American Type Culture Collection (Manassas, VA, USA) and cultured in RPMI 1640 medium, supplemented with $10 \%$ fetal bovine serum (Gibco, Grand Island, NY, USA). Both cell lines were incubated under suitable conditions with $5 \% \mathrm{CO}_{2}\left(37^{\circ} \mathrm{C}\right)$. For hypoxia induction, cells were cultured with $1 \% \mathrm{O}_{2}$ balanced with nitrogen and $5 \% \mathrm{CO}_{2}$.

\section{Quantitative real-time PCR (qRT-PCR) analysis}

QRT-PCR analysis was performed according to the manufacturer's protocol as described previously [23]. GAPDH was used as the reference gene. Primer sequences ( $5^{\prime}$ to $\left.3^{\prime}\right)$ : GLUT1-F:

CTGTGCTCCTGGTTCTGTTCT; GLUT1-R: CAGCTCCTCGGGTGTCTTGT; HK2-F:

GATGGCGTGAACGATGCT; HK2-R: GACGTTGGACGAGATGAGGT; LDHA-F: CAGCCCGATTCCGTTACCTA; LDHA-R: TCAGAGAGACACCAGCAACA; MALAT1-F: GAATTGCGTCATTTAAAGCCTAG; MALAT1-R:

GTTTCATCCTACCACTCCCAATT; HIF-1a-F: ACTAGTGCCACATCATCACC; HIF-1a-R:

ACAGATAACACGTTAGGGCTTC; GAPDH-F: GACGCTGGGGCTGGCATTG; GAPDH-R: GCTGGTGGTCCAGGGGTC.

\section{Western blot analysis}

Western blot analysis was conducted as described previously [24]. In brief, proteins in the cell lysates were electrophoretically separated on sodium dodecyl sulfate polyacrylamide gels and then transferred onto PVDF membranes (Millipore, Burlington, MA, USA). After they were blocked with $5 \%$ skim milk, the blots were incubated with primary antibodies at $4^{\circ} \mathrm{C}$ and secondary antibodies (Aspen, Wuhan, China) at room temperature, followed by visualization with an ECL substrate (Thermo Fisher, Waltham, MA, USA). 
Primary antibodies against HIF-1a, HK2, GLUT1, LDHA, and GAPDH were purchased from Cell Signaling Technology (Danvers, MA, USA).

\section{Glucose uptake and lactate production assays}

SW1990 and PANC-1 cells were seeded into 6-well plates and incubated with different treatments. Glucose uptake and lactate release were detected according to the protocols of a glucose uptake cellbased assay kit (Cayman Chemical, Ann Arbor, MI, USA) and lactic acid assay kit (Nanjing Jiancheng Bio. Nanjing, China), respectively. The production of lactate in each sample was normalized to their respective control groups as indicated.

\section{MTT assay}

The chemosensitivity of pancreatic cancer cells to gemcitabine was measured by the MTT assay, which was described previously [24]. In brief, cells $\left(7 \times 10^{3} /\right.$ well) were seeded into 96-well plates. After different treatments, $20 \mu \mathrm{L}$ of MTT ( $5 \mathrm{mg} / \mathrm{mL}$; Sigma-Aldrich, St. Louis, MO, USA) as incubated per well for $4 \mathrm{~h}$ and subsequently replaced with dimethyl sulfoxide (Sigma-Aldrich). The absorbance values were recorded by an ELISA reader at $490 \mathrm{~nm}$. Each concentration was set up with five replicates.

\section{Apoptosis analysis}

Apoptosis was evaluated with Annexin V/propidium iodide (PI) staining. Briefly, harvested cells were washed in ice-cold PBS and resuspended in binding buffer at a density of $100 \times 10^{4}$ cells. Then, cells were mixed with Annexin V-FITC and PI according to the manufacturer's protocols (KeyGEN Biotech). The fluorescence values for Annexin V-FITC and PI were detected on a flow cytometer (BD Biosciences).

\section{Cell transfection}

SiRNAs targeting MALAT1 and HIF-1 $a$ and negative control siRNA were synthesized by RiboBio Co. (Guangzhou, China). SiRNA transfection was carried out with a mixture of Lipofectamine ${ }^{\mathrm{TM}} 2000$ (Invitrogen) as described previously [23]. The lentiviral vectors with the sequence for Si-MALAT1 (LV-SiMALAT1) and negative control (LV-Si-NC) were purchased from GeneChem (Shanghai, China).

Transfection of the lentiviral vectors into SW1990 cells was conducted according to the manufacturer's protocols. The siRNA sequences ( 5 ' to $3^{\prime}$ ): MALAT1 siRNA: GCAGCCCGAGACUUCUGUA; HIF-1a siRNA: GGAUGGAUUCAUAUUUCUU; NC SIRNA: GGUGUUUCUUUUCUCCCUU.

\section{Chromatin immunoprecipitation (ChIP) assay}

HIF-1a binding to the promoter of MALAT1 was tested using a ChIP assay as described previously [24]. All procedures were carried out according to the protocol of the EZ-ChIPTM Chromatin Immunoprecipitation Kit (Millipore). The Primer sequences ( 5 ' to 3'): Target 1-F:

AGTGCAGTGACAGCGCAGA; Target 1-R: AACCGGCTCTAGCCGGTC; Target 2-F: CGCAGTTGGAGAGACTG; Target 2-R: CGCAAATGGGGATTTGG. 


\section{Tumor xenograft}

The effect of MALAT1 inhibition on gemcitabine chemosensitivity in vivo was examined in a tumor xenograft model. SW1990 cells transfected with LV-Si-MALAT1 were harvested and resuspended in phosphate-buffered saline (PBS). Approximately $5 \times 10^{6}$ cells were subcutaneously injected into the right flanks of male nude mice ( $n=5$; HFK Bioscience Co., Beijing, China). After approximately 5 days, the nude mice were divided into Control, LV-Si-MALAT1, GEM, GEM+LV-Si-MALAT1 groups. For the gemcitabinetreated group, $20 \mathrm{mg} / \mathrm{kg}$ gemcitabine (Selleck.cn, Shanghai, China) was intraperitoneally injected every 3 days. An equal amount of PBS was injected as control. The tumor size was periodically measured and calculated by the following formula: $1 / 2 \times$ length $\times$ width $^{2}$. The mice were euthanized 5 weeks later, and the complete xenograft tumors were excised. Then, the tumor was fixed with $4 \%$ paraformaldehyde, embedded in paraffin and cut into $5 \mu \mathrm{m}$ sections. The proliferative activity of tumor cells was determined by Ki-67 immunohistochemical staining, while the apoptosis level was measured by TUNEL immunofluorescent staining.

\section{Statistical Analysis}

The data are presented as the means \pm SD. Comparisons between 2 groups were conducted with Student's t-test. SPSS version 18.0 was used for data analysis. $\mathrm{P}<0.05$ was considered to be the threshold of significance.

\section{Results}

\section{Enhanced glycolytic flux induced by gemcitabine promotes the chemoresistance of pancreatic cancer cells}

Aerobic glycolysis, a hallmark of pancreatic cancer, has been shown to closely correlate with drug resistance [5]. The previous results have revealed that low-dose gemcitabine treatment increases acquired chemoresistance via stemness induction [24]; thus, we next detected the change in glycolytic activity upon gemcitabine treatment. As shown in Fig. $1 \mathrm{a}$ and $\mathrm{b}$, a low dose of gemcitabine upregulated both the mRNA and protein expression levels of key enzymes in glycolytic flux, such as GLUT1, HK2, and LDHA. Notably, an increase in glucose uptake, as indicated by the fluorescent deoxyglucose analog 2-NBDG, was observed after gemcitabine treatment in both pancreatic cancer cell lines (Fig. 1C and d). Furthermore, gemcitabine significantly enhanced the release of lactate into culture medium (Fig. 1e).

To investigate the effect of enhanced aerobic glycolysis on gemcitabine resistance, we utilized 2-DG, a glucose analog, to competitively inhibit glycolysis. As shown in Fig. 1f, 2-DG effectively inhibited lactate production in both cell lines. Moreover, suppression of glycolysis dramatically promoted gemcitabineinduced apoptosis of pancreatic cancer cells (Fig. 1g and h). Accordingly, the killing effect of gemcitabine was also reinforced when combined with 2-DG treatment (Fig. 1i). Taken together, these data suggest that enhanced glycolytic flux induced by a low dose of gemcitabine promotes acquired chemoresistance in pancreatic cancer cells. 


\section{MALAT1 partially mediates gemcitabine-induced glycolysis and chemoresistance}

MALAT1 has been reported to promote the malignancy of various types of cancer, including pancreatic cancer [25-27]. The expression of MALAT1 was indicated to be inversely correlated with the gemcitabine response rate in pancreatic cancer [19]. We first determined the change in MALAT1 expression upon gemcitabine treatment. As shown in Fig. 2a, a low dose of gemcitabine increased the mRNA level of MALAT1 in a dose-dependent manner. To further investigate the role of MALAT1 in gemcitabine resistance, RNA interference targeting MALAT1 was employed. QRT-PCR analysis revealed that MALAT1targeted siRNA could effectively silence the expression of MALAT1 (Fig. 2b). Accordingly, gemcitabineinduced MALAT1 expression was also inhibited (Fig. 2c). In addition, inhibition of MALAT1 significantly reduced both the mRNA and protein levels of the key glycolytic enzymes GLUT1, HK2, and LDHA induced by gemcitabine (Fig. 2d and e). Glucose uptake and lactate production were also attenuated (Additional file 1: Figure S1a-c). Furthermore, gemcitabine-induced apoptosis was reinforced after MALAT1 knockdown in both cell lines (Fig. $2 \mathrm{f}$ and $\mathrm{g}$ ). Consistent with these changes, silencing MALAT1 significantly enhanced the sensitivity of both tested cell lines to gemcitabine (Fig. 2h). Altogether, our results suggest that MALAT1 partially mediates gemcitabine-induced glycolysis and chemoresistance.

\section{HIF-1apartially mediates gemcitabine-induced glycolysis and chemoresistance}

Activation of HIF-1a is considered an important upstream mediator of glycolysis. A previous report verified that gemcitabine could promote the activation of HIF-1a [28]. We further tested whether HIF-1a mediated gemcitabine-induced glycolysis. As shown in Fig. 3a, silencing HIF-1a significantly reduced its expression in both tested cell lines, which resulted in dramatic reductions in both the mRNA and protein levels of GLUT1, HK2, and LDHA induced by gemcitabine (Fig. 3b-e). Moreover, HIF-1a knockdown obviously attenuated gemcitabine-enhanced lactate secretion in the culture medium (Fig. 3f). Notably, suppression of HIF-1 a further facilitated apoptosis induced by gemcitabine in both cancer cell lines (Fig. $3 \mathrm{~g}$ and $\mathrm{h}$ ). Altogether, our data suggest that HIF-1 a partially participates in gemcitabine-induced glycolysis and chemoresistance.

\section{Gemcitabine facilitated the reciprocal loop of MALAT1 and HIF-1a}

It has been reported that MALAT1 could stabilize HIF-1a in a normal human liver cell line [21]. In our present study, we showed that silencing MALAT1 also significantly inhibited the protein level of HIF-1a in both pancreatic cancer lines. In addition, after MALAT1 knockdown, gemcitabine-induced HIF-1a expression was attenuated (Fig. 4a). We next tested the effect of HIF-1a expression on gemcitabineinduced MALAT1 expression, and a similar obvious reduction in MALAT1 level induced by gemcitabine was observed after HIF-1a inhibition (Fig. 4b). To further elucidate the regulatory mechanism of HIF-1a on the expression of MALAT1, ChIP analysis was performed. Based on the prediction by JASPAR software, there were several putative HIF-1a binding sites on the promoter region of MALAT1 (Fig. 4d). We initiated hypoxia to activate HIF-1 $a$ and observed that the mRNA level of MALAT1 was significantly upregulated (Fig. 4c). Furthermore, enhanced binding of HIF-1a to the promoter of MALAT1 was observed after 
hypoxia treatment (Fig. 4e). Taken together, our results suggest that MALAT1 forms a positive feedback loop with HIF-1a, which was reinforced by gemcitabine treatment.

\section{MALAT1 inhibition enhances the killing effect of gemcitabine in vivo}

The in vivo effect of the combination of MALAT1 inhibition and gemcitabine chemotherapy was further examined in a xenograft model, which was established by subcutaneously injecting tumor cells into the right flanks of nude mice. As shown in Fig. 5a-c, compared with the control group, the LV-Si-MALAT1 group showed significantly inhibition of the xenograft growth rate and size, as well as the tumor weight. When combined with gemcitabine chemotherapy, these effects were more remarkable. Next, we detected tumor cell proliferation and apoptotic markers by using immunofluorescence and immunohistochemical analysis. The data showed that gemcitabine combined with LV-Si-MALAT1 transfection dramatically reduced the Ki-67 index and increased TUNEL staining compared with those of the control group (Fig. $5 d)$. Altogether, our results demonstrated that MALAT1 inhibition enhanced the apoptosis and killing effect induced by gemcitabine in vivo.

\section{Discussion}

For pancreatic cancer, gemcitabine has been approved as a first-line drug for decades, especially in patients with advanced and metastatic disease. However, the clinical response rate was dismal, with fewer than $20 \%$ patients exhibiting an improved response. Due to profound inherent or acquired chemoresistance [29], the results of combined treatment or adjuvant drugs were also unsatisfactory. Aerobic glycolysis, a hallmark of tumors, has been shown to promote the malignant phenotypes of pancreatic cancer, including drug resistance. In the present study, we found a new mechanism of acquired resistance to gemcitabine through the induction of glycolysis. In addition, the positive feedback loop of MALAT1 and HIF-1a induced by gemcitabine mediated this biological effect (Fig. 6). Inhibition of MALAT1 expression synergistically enhanced the killing effect of gemcitabine in vivo. Thus, our study presents new insight into gemcitabine chemoresistance.

Accumulated evidence indicates that aerobic glycolysis facilitates cancer cell survival and resistance to apoptosis induced by chemotherapy [30]. Inhibition of glycolysis has been suggested to exert a potential therapeutic effect in pancreatic cancer [31,32]. In our present study, we showed that a low concentration of gemcitabine promoted glycolytic flux, exhibiting upregulated key glycolytic enzymes, glucose consumption and lactate production in the culture medium. These findings of enhanced glycolysis might further reinforce acquired gemcitabine resistance and explain the worsening response during the course of treatment. In addition, our data revealed that concomitant glycolysis inhibition partially recovered gemcitabine-induced apoptosis and chemosensitivity. On the other hand, we observed that a relatively high dose of gemcitabine did not induce the expression of key glycolytic enzymes (data not shown). A similar effect was also found on the induction of stemness in pancreatic cancer [24], since glycolysis has been shown to promote the stemness phenotype in gemcitabine-resistant pancreatic cancer cells [33]. 
Collectively, our data suggest an undesired mechanism of acquired gemcitabine resistance by inducing glycolysis.

It has been reported that MALAT1 is overexpressed and promotes malignancy in various types of cancer, including pancreatic cancer [17, 34-36]. In our study, we showed that MALAT1 expression could be induced by gemcitabine. In addition, inhibition of MALAT1 expression enhanced gemcitabine-induced apoptosis and the killing effects of gemcitabine in vitro and in vivo. This suggested that MALAT1 plays a promoting role in driving gemcitabine resistance, which is in line with a previous report on the inverse correlation between MALAT1 levels and both gemcitabine response and PFS in patients with pancreatic cancer [19]. MALAT1 has been shown to enhance glycolysis in hepatocellular carcinoma [20]. In our present study, we revealed that MALAT1 inhibition blocked the activities of gemcitabine-enhanced glycolytic enzymes, glucose consumption and lactate production. Altogether, our results indicated that MALAT1 partially mediated gemcitabine-induced glycolysis and chemoresistance in pancreatic cancer.

HIF-1a is considered a master regulator driving glycolytic metabolism in cancer cells [37, 38]. Moreover, gemcitabine resistance was suggested to be associated with HIF-1a induction in gemcitabine-resistant pancreatic cancer cells [39]. In our study, we showed that gemcitabine promoted the expression of HIF-1a, while knockdown of HIF-1a inhibited gemcitabine-induced glycolysis and facilitated apoptosis of cancer cells. Our results suggested the promoting role of HIF-1a in glycolysis and chemoresistance induced by gemcitabine. In addition, we observed that MALAT1 knockdown inhibited the activation of HIF-1a induced by gemcitabine, which was similar to the findings in previously published data [21, 40]. Intriguingly, after silencing HIF-1a, gemcitabine-induced MALAT1 expression was also attenuated. HIF-1a is the key effector activated under hypoxia [41]. Our study verified that HIF-1a occupied the presumed promoter region of MALAT1, and this occupation was enhanced by hypoxia treatment. Therefore, our data suggested that the reciprocal regulation between MALAT1 and HIF-1a mediates gemcitabine-induced glycolysis and chemoresistance.

\section{Conclusions}

Our results identify a new mechanism of acquired gemcitabine resistance by inducing glycolysis in pancreatic cancer. In addition, the positive feedback loop between MALAT1 and HIF-1a enhanced by gemcitabine partially mediates this effect. Our findings might provide novel insights into chemoresistance and novel potential targets for combating pancreatic cancer.

\section{Abbreviations}

ATP: adenosine triphosphate; LncRNAs: long non-coding RNAs; MALAT1: metastasis-associated lung adenocarcinoma transcript 1; PFS: progression-free-survival; HIF-1a: hypoxia inducible factor 1a; GEM: gemcitabine; PBS: phosphate-buffered saline.

\section{Declarations}




\section{Ethics approval and consent to participate}

This study was approved by the ethical review board of Renmin Hospital of Wuhan University (Wuhan, China).

\section{Consent for publication}

Not applicable.

\section{Availability of data and materials}

The datasets used or analysed during the study are available from the corresponding author on reasonable request.

\section{Competing interests}

The authors declare that they have no competing interests.

\section{Funding}

This study was supported by grants from the National Natural Science Foundation of China (No.81803030) and the Scientific Research Foundation of Wuhan University (No.2042018kf0118).

\section{Authors' contributions}

JT was responsible for the conceptualization and supervision of experiments. ZLZ, HH, and QG carried out the experiments, analyzed the data, and wrote the draft. YPR, HJL and ZCZ performed parts of the experiments. PM and CBZ participated in data collection. ZGT participated in revising and polishing the manuscript. All authors have read and approved the final version of manuscript.

\section{Acknowledgements}

Not applicable.

\section{References}

1. Jemal A, Bray F, Center MM, Ferlay J, Ward E, Forman D. Global cancer statistics. CA Cancer J Clin. 2011;61:69-90.

2. Vander Heiden MG, Cantley LC, Thompson CB. Understanding the Warburg effect: the metabolic requirements of cell proliferation. Science. 2009;324:1029-33.

3. Yan L, Raj P, Yao W, Ying H. Glucose Metabolism in Pancreatic Cancer. Cancers (Basel). 2019;11:1460.

4. Ma L, Zong X. Metabolic Symbiosis in Chemoresistance: Refocusing the Role of Aerobic Glycolysis. Front Oncol. 2020;10:5. 
5. Dai S, Peng Y, Zhu Y, Xu D, Zhu F, Xu W, et al. Glycolysis promotes the progression of pancreatic cancer and reduces cancer cell sensitivity to gemcitabine. Biomed Pharmacother. 2020;121:109521.

6. Zhao H, Wu S, Li H, Duan Q, Zhang Z, Shen Q, et al. ROS/KRAS/AMPK Signaling Contributes to Gemcitabine-Induced Stem-like Cell Properties in Pancreatic Cancer. Mol Ther Oncolytics. 2019;14:299-312.

7. Moschovis D, Gazouli M, Tzouvala M, Vezakis A, Karamanolis G. Long non-coding RNA in pancreatic adenocarcinoma and pancreatic neuroendocrine tumors. Ann Gastroenterol. 2017;30:622-8.

8. Jin KT, Lu ZB, Lv JQ, Zhang JG. The role of long non-coding RNAs in mediating chemoresistance by modulating autophagy in cancer. RNA Biol. 2020;17:1727-40.

9. Chi HC, Tsai CY, Tsai MM, Yeh CT, Lin KH. Roles of Long Noncoding RNAs in Recurrence and Metastasis of Radiotherapy-Resistant Cancer Stem Cells. Int J Mol Sci. 2017;18:1903.

10. Yang Q, Li K, Huang X, Zhao C, Mei Y, Li X, et al. IncRNA SLC7A11-AS1 Promotes Chemoresistance by Blocking SCF(beta-TRCP)-Mediated Degradation of NRF2 in Pancreatic Cancer. Mol Ther Nucleic Acids. 2020;19:974-85.

11. Wang L, Dong P, Wang W, Huang M, Tian B. Gemcitabine treatment causes resistance and malignancy of pancreatic cancer stem-like cells via induction of IncRNA HOTAIR. Exp Ther Med. 2017; 14:4773-80.

12. Hutchinson JN, Ensminger AW, Clemson CM, Lynch CR, Lawrence JB, Chess A. A screen for nuclear transcripts identifies two linked noncoding RNAs associated with SC35 splicing domains. BMC Genomics. 2007;8:39.

13. Ji P, Diederichs S, Wang W, Boing S, Metzger R, Schneider PM, et al. MALAT-1, a novel noncoding RNA, and thymosin beta4 predict metastasis and survival in early-stage non-small cell lung cancer. Oncogene. 2003;22:8031-41.

14. Chen Q, Zhu C, Jin Y. The Oncogenic and Tumor Suppressive Functions of the Long Noncoding RNA MALAT1: An Emerging Controversy. Front Genet. 2020;11:93.

15. Sun Y, Ma L. New Insights into Long Non-Coding RNA MALAT1 in Cancer and Metastasis. Cancers (Basel). 2019;11:216.

16. Liu JH, Chen G, Dang YW, Li CJ, Luo DZ. Expression and prognostic significance of IncRNA MALAT1 in pancreatic cancer tissues. Asian Pac J Cancer Prev. 2014;15:2971-7.

17. Jiao F, Hu H, Yuan C, Wang L, Jiang W, Jin Z, et al. Elevated expression level of long noncoding RNA MALAT-1 facilitates cell growth, migration and invasion in pancreatic cancer. Oncol Rep. 2014;32:2485-92.

18. Jiao F, Hu H, Han T, Yuan C, Wang L, Jin Z, et al. Long noncoding RNA MALAT-1 enhances stem celllike phenotypes in pancreatic cancer cells. Int J Mol Sci. 2015;16:6677-93.

19. Wang CJ, Shi SB, Tian J, Xu J, Niu ZX. IncRNA MALAT1, HOTTIP and PVT1 as predictors for predicting the efficacy of GEM based chemotherapy in first-line treatment of pancreatic cancer patients. Oncotarget. 2017;8:95108-15. 
20. Malakar P, Stein I, Saragovi A, Winkler R, Stern-Ginossar N, Berger M, et al. Long Noncoding RNA MALAT1 Regulates Cancer Glucose Metabolism by Enhancing mTOR-Mediated Translation of TCF7L2. Cancer Res. 2019;79:2480-93.

21. Luo F, Liu X, Ling M, Lu L, Shi L, Lu X, et al. The IncRNA MALAT1, acting through HIF-1alpha stabilization, enhances arsenite-induced glycolysis in human hepatic L-02 cells. Biochim Biophys Acta. 2016;1862:1685-95.

22. Liu H, Zhang Z, Xiong W, Zhang L, Du Y, Liu Y, et al. Long non-coding RNA MALAT1 mediates hypoxia-induced pro-survival autophagy of endometrial stromal cells in endometriosis. J Cell Mol Med. 2019;23:439-52.

23. Yin T, Zhang Z, Cao B, Duan Q, Shi P, Zhao H, et al. Bmi1 inhibition enhances the sensitivity of pancreatic cancer cells to gemcitabine. Oncotarget. 2016;7:37192-204.

24. Zhang Z, Duan Q, Zhao H, Liu T, Wu H, Shen Q, et al. Gemcitabine treatment promotes pancreatic cancer stemness through the Nox/ROS/NF-kappaB/STAT3 signaling cascade. Cancer Lett. 2016;382:53-63.

25. Cao Y, Zhang F, Wang H, Bi C, Cui J, Liu F, et al. LncRNA MALAT1 mediates doxorubicin resistance of hepatocellular carcinoma by regulating miR-3129-5p/Nova1 axis. Mol Cell Biochem. 2020; doi: 10.1007/s11010-020-03904-6.

26. Wang P, Hu L, Fu G, Lu J, Zheng Y, Li Y, et al. LncRNA MALAT1 Promotes the Proliferation, Migration, and Invasion of Melanoma Cells by Downregulating miR-23a. Cancer Manag Res. 2020;12:6553-62.

27. Cheng Y, Imanirad P, Jutooru I, Hedrick E, Jin UH, Rodrigues Hoffman A, et al. Role of metastasisassociated lung adenocarcinoma transcript-1 (MALAT-1) in pancreatic cancer. PLoS One. 2018;13:e0192264.

28. Arora S, Bhardwaj A, Singh S, Srivastava SK, McClellan S, Nirodi CS, et al. An undesired effect of chemotherapy: gemcitabine promotes pancreatic cancer cell invasiveness through reactive oxygen species-dependent, nuclear factor kappaB- and hypoxia-inducible factor 1alpha-mediated upregulation of CXCR4. J Biol Chem. 2013;288:21197-207.

29. de Sousa Cavalcante L, Monteiro G. Gemcitabine: metabolism and molecular mechanisms of action, sensitivity and chemoresistance in pancreatic cancer. Eur J Pharmacol. 2014;741:8-16.

30. Icard P, Shulman S, Farhat D, Steyaert JM, Alifano M, Lincet H. How the Warburg effect supports aggressiveness and drug resistance of cancer cells? Drug Resist Updat. 2018;38:1-11.

31. Isayev O, Rausch V, Bauer N, Liu L, Fan P, Zhang Y, et al. Inhibition of glucose turnover by 3bromopyruvate counteracts pancreatic cancer stem cell features and sensitizes cells to gemcitabine. Oncotarget. 2014;5:5177-89.

32. Kim DJ, Park YS, Kang MG, You YM, Jung Y, Koo H, et al. Pyruvate kinase isoenzyme M2 is a therapeutic target of gemcitabine-resistant pancreatic cancer cells. Exp Cell Res. 2015;336:119-29.

33. Zhao H, Duan Q, Zhang Z, Li H, Wu H, Shen Q, et al. Up-regulation of glycolysis promotes the stemness and EMT phenotypes in gemcitabine-resistant pancreatic cancer cells. J Cell Mol Med. 2017;21:2055-67. 
34. Fan L, Huang X, Chen J, Zhang K, Gu YH, Sun J, et al. Long non-coding RNA MALAT1 contributes to sorafenib resistance by targeting miR-140-5p/Aurora-A signaling in hepatocellular carcinoma. Mol Cancer Ther. 2020:1197-1209.

35. Ji Q, Zhang L, Liu X, Zhou L, Wang W, Han Z, et al. Long non-coding RNA MALAT1 promotes tumour growth and metastasis in colorectal cancer through binding to SFPQ and releasing oncogene PTBP2 from SFPQ/PTBP2 complex. Br J Cancer. 2014;111:736-48.

36. Wang QM, Lian GY, Song Y, Huang YF, Gong Y. LncRNA MALAT1 promotes tumorigenesis and immune escape of diffuse large B cell lymphoma by sponging miR-195. Life Sci. 2019;231:116335.

37. Marin-Hernandez A, Gallardo-Perez JC, Ralph SJ, Rodriguez-Enriquez S, Moreno-Sanchez R. HIF1 alpha modulates energy metabolism in cancer cells by inducing over-expression of specific glycolytic isoforms. Mini Rev Med Chem. 2009;9:1084-101.

38. Yu L, Chen X, Wang L, Chen S. The sweet trap in tumors: aerobic glycolysis and potential targets for therapy. Oncotarget. 2016;7:38908-26.

39. Wang R, Cheng L, Xia J, Wang Z, Wu Q, Wang Z. Gemcitabine resistance is associated with epithelialmesenchymal transition and induction of HIF-1alpha in pancreatic cancer cells. Curr Cancer Drug Targets. 2014;14:407-17.

40. Liu XQ, Duan LS, Chen YQ, Jin XJ, Zhu NN, Zhou X, et al. IncRNA MALAT1 Accelerates Wound Healing of Diabetic Mice Transfused with Modified Autologous Blood via the HIF-1alpha Signaling Pathway. Mol Ther Nucleic Acids. 2019;17:504-15.

41. Tirpe AA, Gulei D, Ciortea SM, Crivii C, Berindan-Neagoe I. Hypoxia: Overview on Hypoxia-Mediated Mechanisms with a Focus on the Role of HIF Genes. Int J Mol Sci. 2019;20:6140.

\section{Figures}



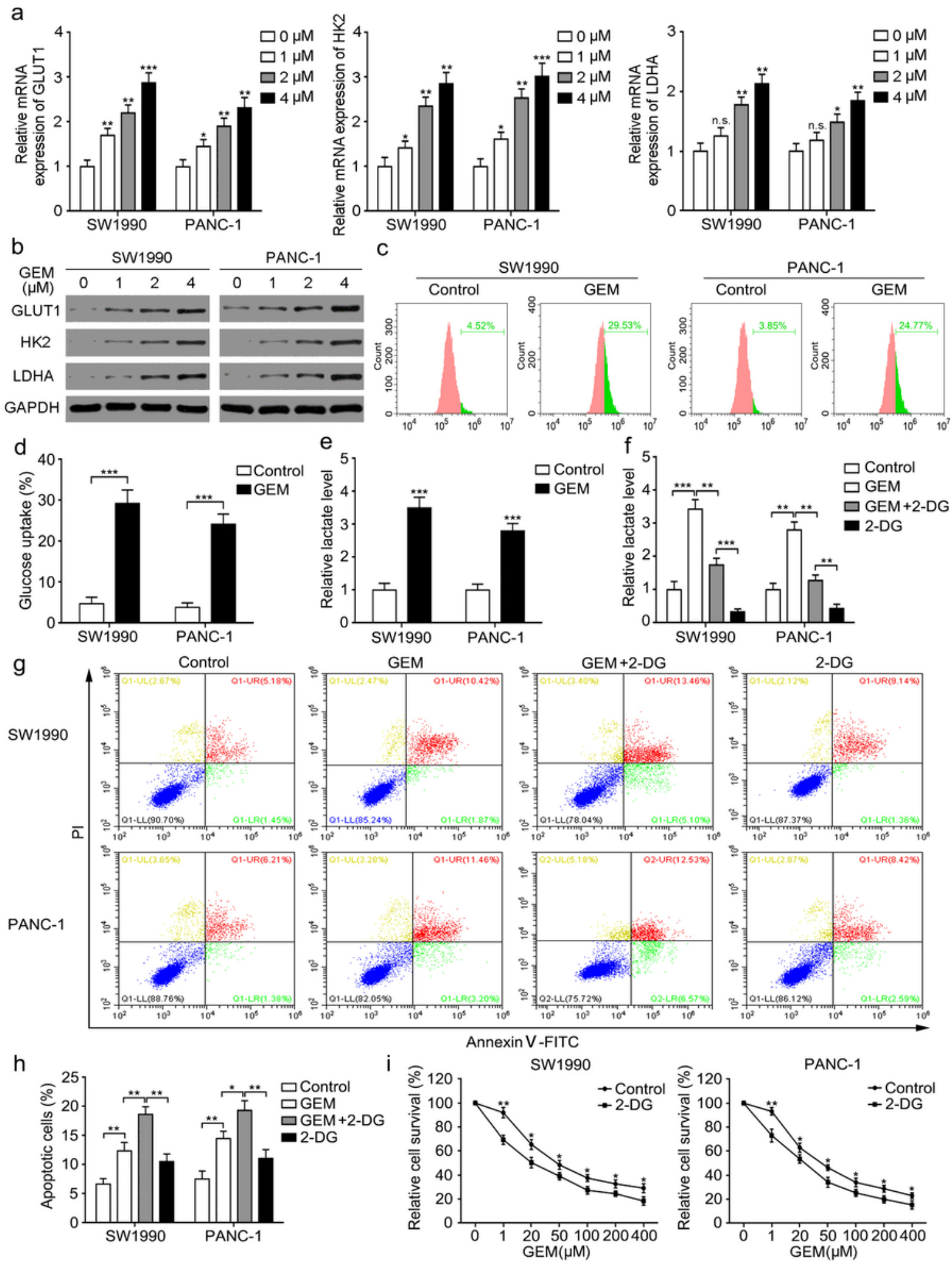

\section{Figure 1}

$<p>$ Enhanced glycolytic flux induced by gemcitabine promotes the chemoresistance of pancreatic cancer cells. <strong $>\mathrm{a}<$ /strong $>$ After treatment with different concentrations of gemcitabine for $24 \mathrm{~h}$, the mRNA expression levels of GLUT1, HK2, and LDHA were analyzed by qRT-PCR. <strong $>b</$ strong $>$ The protein levels of GLUT1, HK2, and LDHA were measured by western blot analysis after the above treatments. $<$ strong $>$ c, $d</$ strong $>$ Two pancreatic cancer cell lines were treated with 4 \&mu;M gemcitabine for 24 h, 
and glucose uptake was tested by the glucose uptake assay: <strong $>c</$ strong $>$ Representative image of 2-NBDG uptake in cancer cells; $<$ strong $>\mathrm{d}</$ strong $>$ The change in relative glucose uptake ability. $<$ strong $>\mathrm{e}<$ /strong $>$ Lactate production was detected by the lactate production assay after treatment as indicated above. SW1990 and PANC-1 cells were pretreated with 5 mM 2-DG for $1 \mathrm{~h}$, followed by gemcitabine treatment. $<$ strong $>\mathrm{f}</$ strong $>$ The relative lactate level was measured by the lactate production assay. $<$ strong $>$ g, $h</$ strong $>$ The apoptosis of pancreatic cancer cells was detected by using the Annexin V-FITC/PI method: <strong $>$ g $</$ strong $>$ Representative image of apoptotic cells; $<$ strong $>\mathrm{h}</$ strong $>$ The percentage of apoptotic cancer cells was analyzed by counting the number of events in the right upper and right lower quadrants. <strong>i</strong > After pretreatment with 2-DG, the sensitivity of the two cancer cell lines to gemcitabine treatment was measured by the MTT assay. The results shown are from three independent experiments. $<$ em $>$ n.s. $</$ em $>$ no significance; ${ }^{*}<e m>P</ e m>\& \mid t$; $0.05 ; * *<e m>P</ e m>\&|t ; 0.01 ; * \star *<e m>P</ e m>\&| t ; 0.001 .</ p>$ 
a

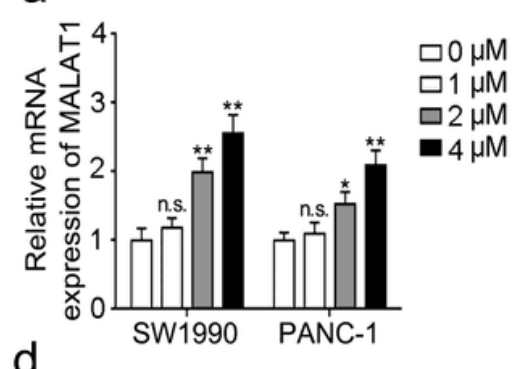

$\mathrm{b}$

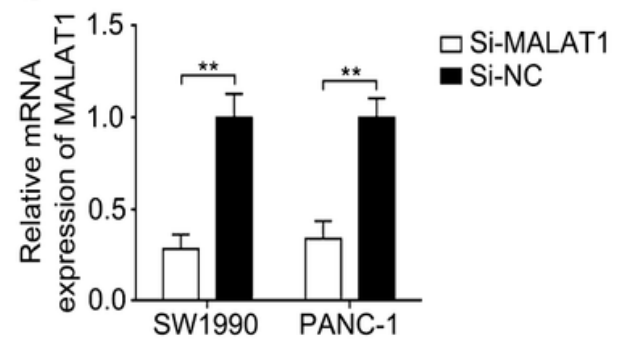

C

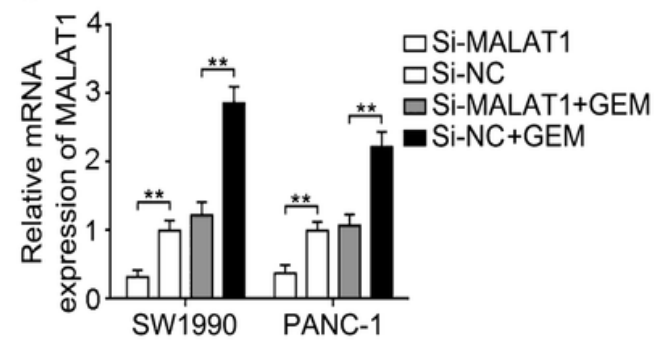

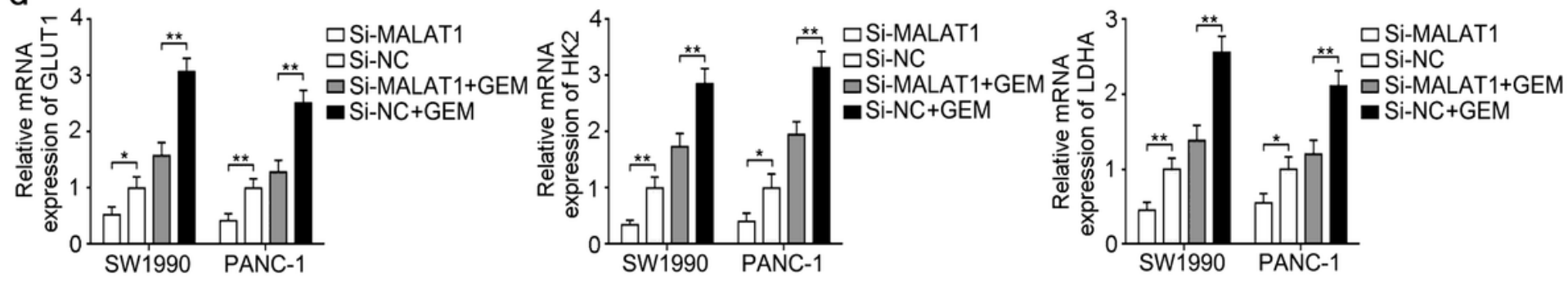

e
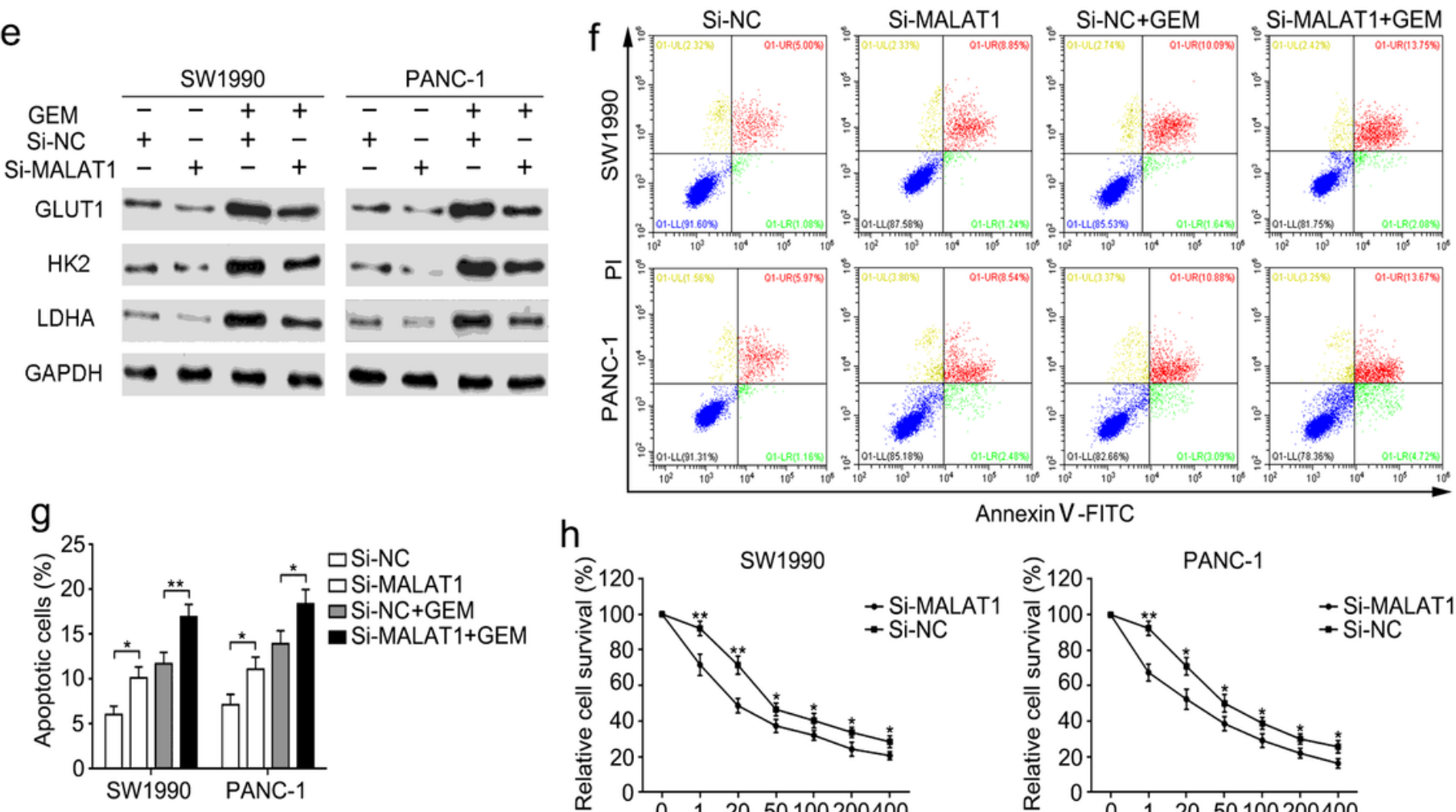

$\mathrm{h}$

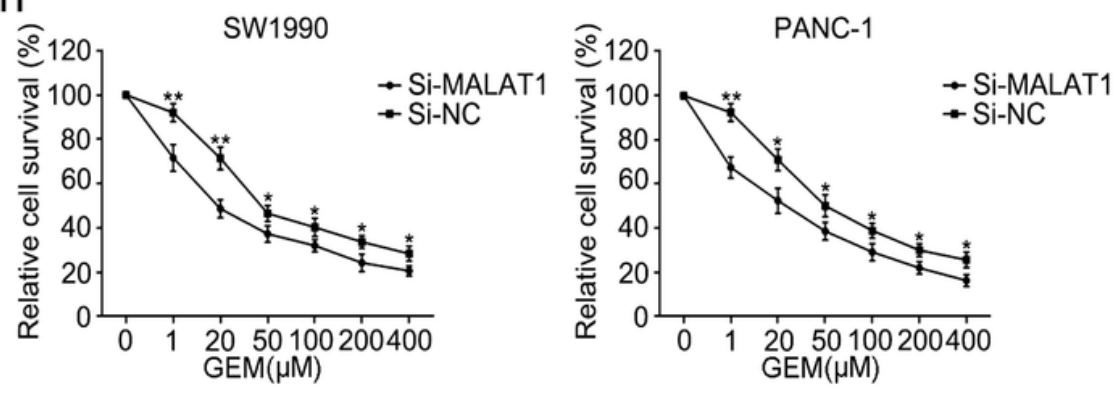

Figure 2

<p>MALAT1 partially mediates gemcitabine-induced glycolysis and chemoresistance. $<$ strong $>$ a $</$ strong $>$ The mRNA level of MALAT1 in two cancer cell lines was analyzed by qRT-PCR after treatment with different concentrations of gemcitabine. <strong $>b</$ strong $>$ qRT-PCR analysis was used to detect the knockdown efficiency of siRNA targeting MALAT1. SW1990 and PANC-1 cells were transfected with siMALAT1 or si-NC, followed by gemcitabine treatment. <strong $>c$, $d</$ strong $>$ The mRNA expression levels of MALAT1, GLUT1, HK2, and LDHA were measured by qRT-PCR analysis. <strong>e</strong > The protein levels of GLUT1, HK2, and LDHA were detected by western blot analysis. <strong $>\mathrm{f}, \mathrm{g}</$ strong $>$ The apoptosis of pancreatic cancer cells was detected by using the Annexin V-FITC/PI method: 
$<$ strong $>\mathrm{f}</$ strong $>$ Representative dot plots of apoptotic cells; $<$ strong $>\mathrm{g}</$ strong $>$ Statistical analysis of apoptotic cells. $<$ strong $>\mathrm{h}</$ strong $>$ After transfection with si-MALAT1 or si-NC, the sensitivity of the two cell lines to gemcitabine was measured by the MTT assay. The data shown are representative of three independent assays. $<$ em>n.s. $</$ em $>$ no significance; ${ }^{*}<\mathrm{em}>\mathrm{P}</ \mathrm{em}>\&|\mathrm{t} ; 0.05 ; * \star<e m>\mathrm{P}</ \mathrm{em}>\&| \mathrm{lt} ; 0.01$. $</ p>$
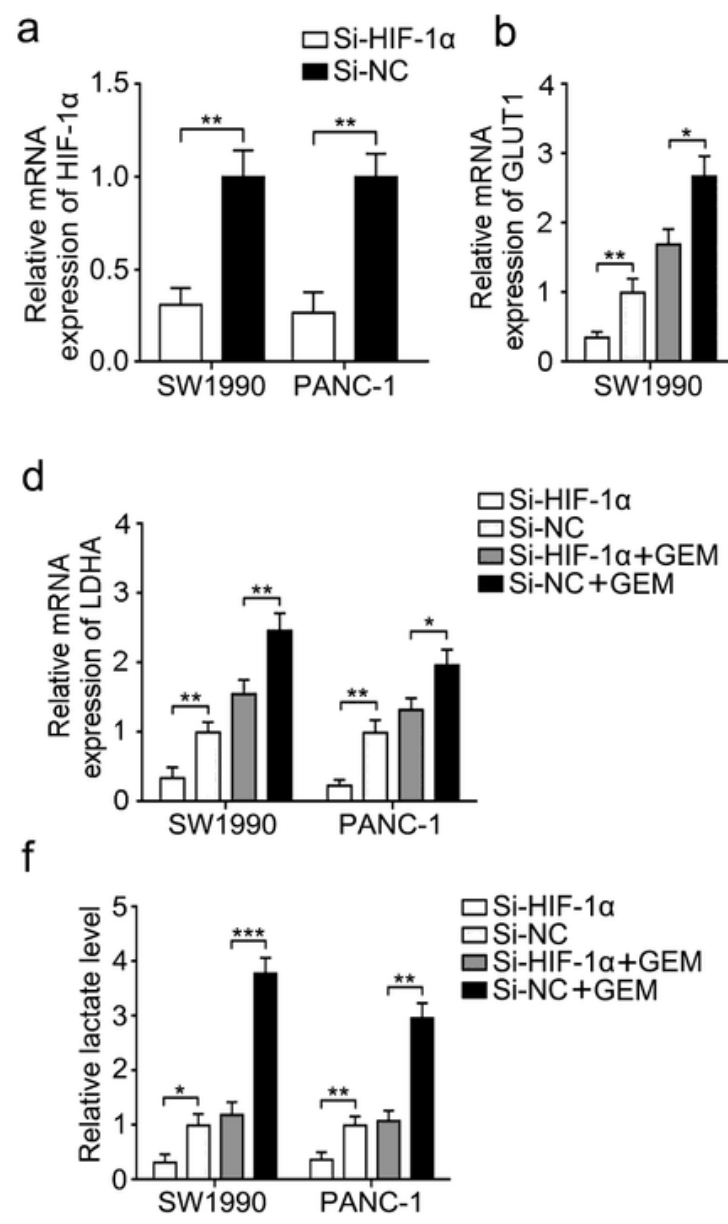

h

DSi-HIF-1a C DSi-HIF-1a
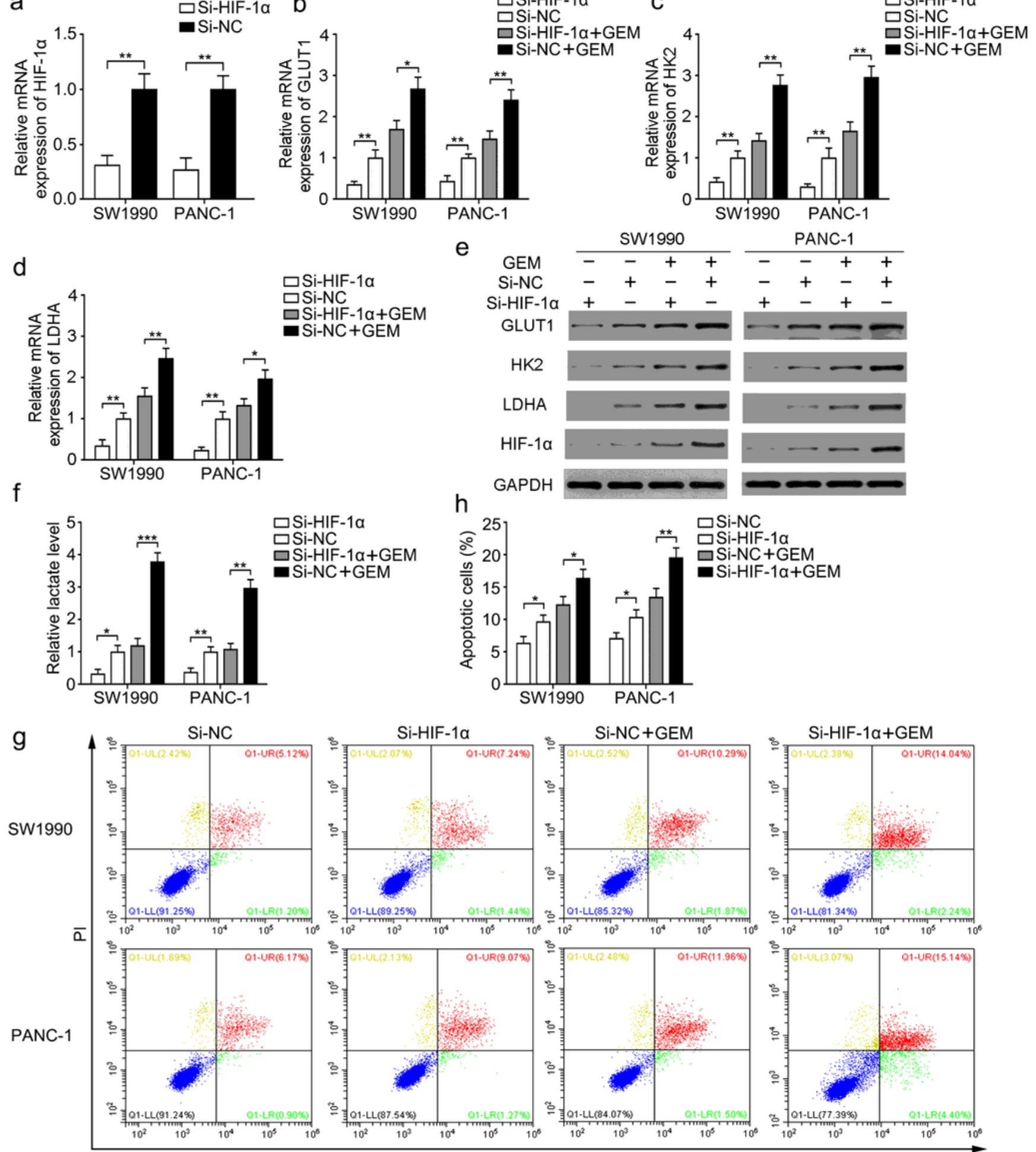

Annexin V-FITC

Figure 3 
HIF-1 a partially mediates gemcitabine-induced glycolysis and chemoresistance. a qRT-PCR analysis was used to determine the efficiency of silencing HIF-1a. SW1990 and PANC-1 cells were transfected with siHIF-1a or si-NC, followed by gemcitabine treatment. b, c, d The mRNA levels of GLUT1, HK2, and LDHA were detected by qRT-PCR. e The protein levels of GLUT1, HK2, and LDHA were detected by western blot analysis. $f$ The relative lactate levels were measured by the lactate release assay. $g, h$ The changes in apoptosis rates in the two cancer cell lines: $g$ Representative apoptotic dot plots; $h$ Statistical analysis of apoptotic status. The results shown are from three independently repeated assays. ${ }^{*} P<0.05$; ${ }^{*} P<0.01$; $* * * P<0.001$.

a

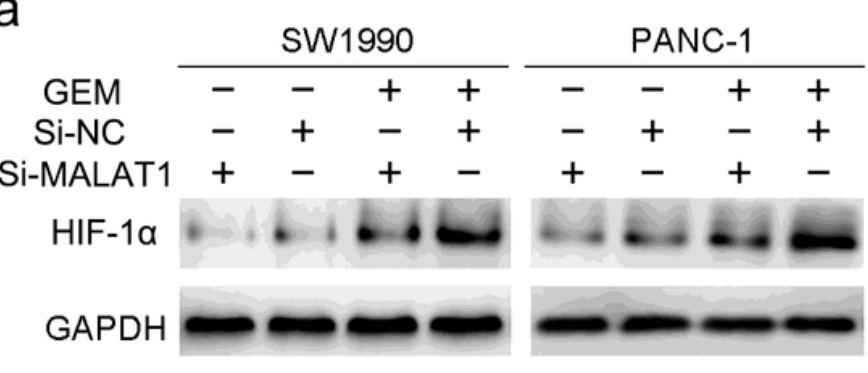

d

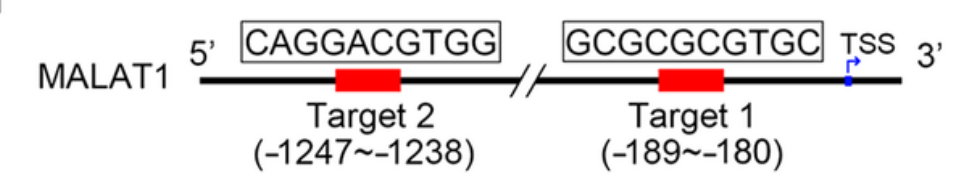

e

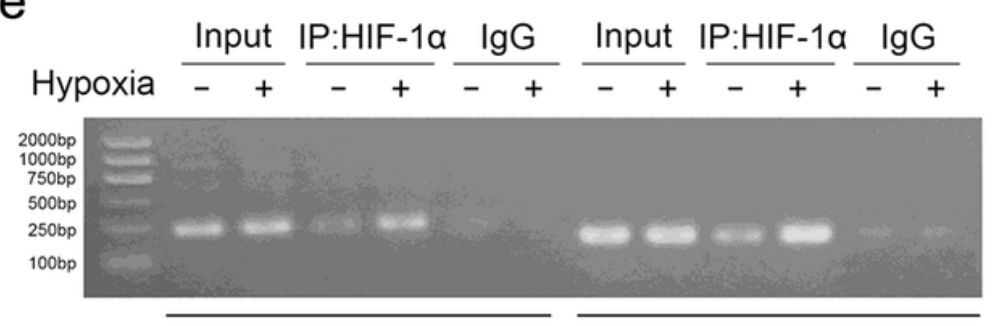

Target1

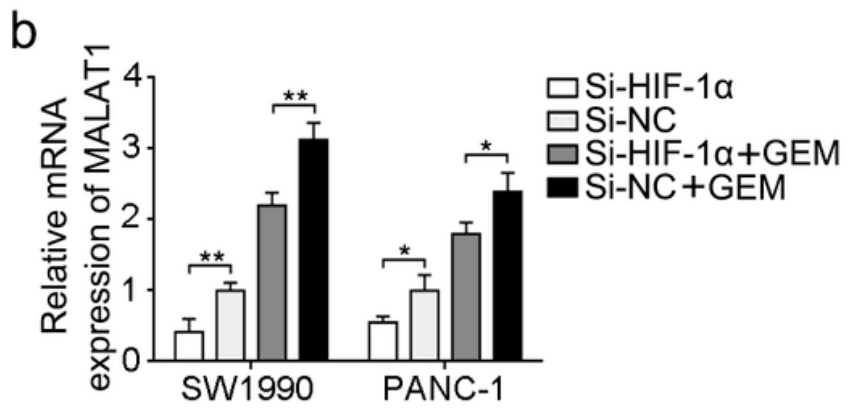

C

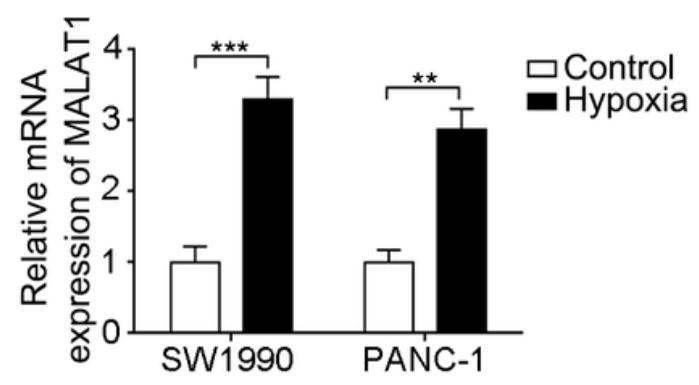

\section{Figure 4}

Gemcitabine facilitated the reciprocal positive feedback loop of MALAT1 and HIF-1a. a SW1990 and PANC-1 cells were transfected with si-MALAT1 or si-NC, followed by gemcitabine treatment, and the protein level of HIF-1a was measured by western blot analysis. b Two cell lines were transfected with siHIF-1a or si-NC, followed by gemcitabine treatment, and the mRNA level of MALAT1 was detected by qRTPCR analysis. c The mRNA expression of MALAT1 was measured by qRT-PCR analysis after exposure to hypoxia treatment for $9 \mathrm{~h}$. $d$ The putative binding sites of HIF-1a to the promoter of MALAT1 are shown. e After hypoxia treatment, a ChIP assay was used to determine the binding abilities at each site. The results shown are from three independent experiments. ${ }^{*} \mathrm{P}<0.05 ;{ }^{* \star} \mathrm{P}<0.01 ; * \star * P<0.001$. 
a

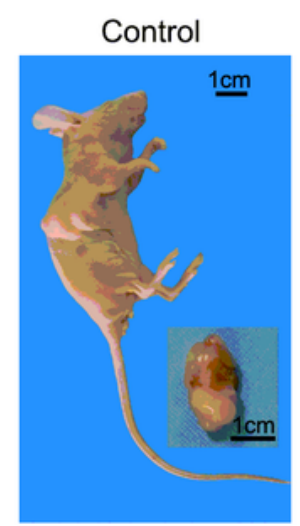

LV-Si-MALAT1
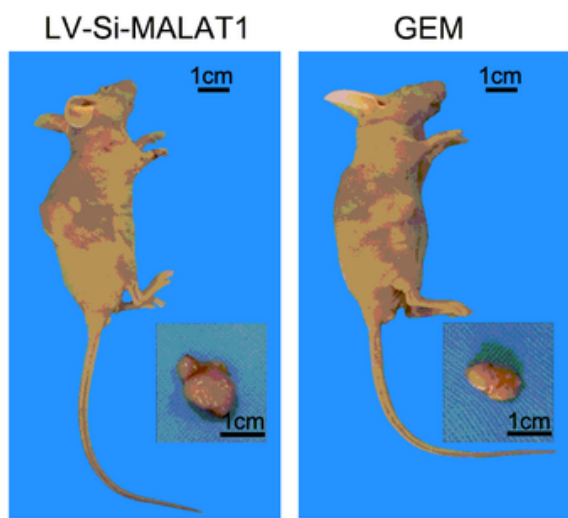

GEM+LV-Si-MALAT1

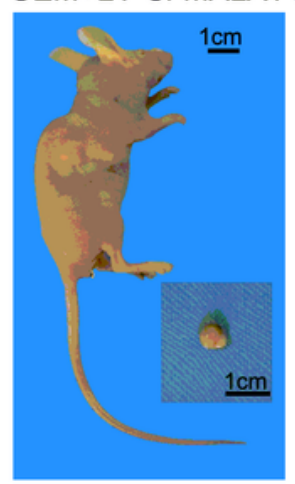

b

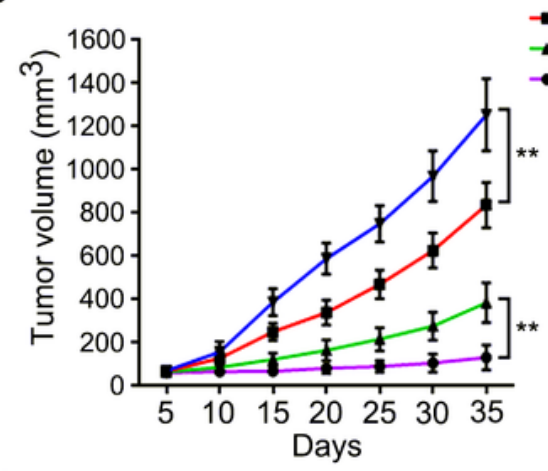

d

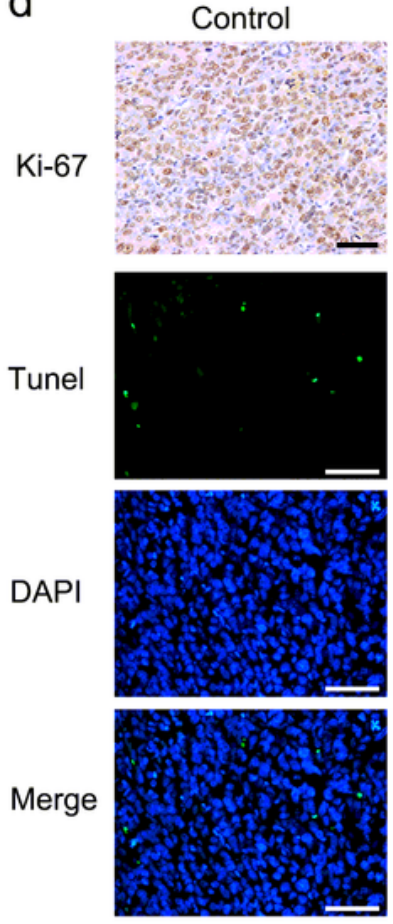

LV-Si-MALAT1
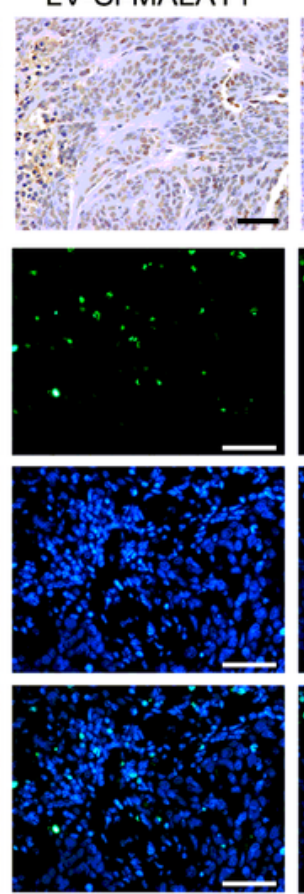

- LV-Si-MALAT1

- GEM

$\rightarrow$ GEM+LV-Si-MALAT1

C $\square$ Control
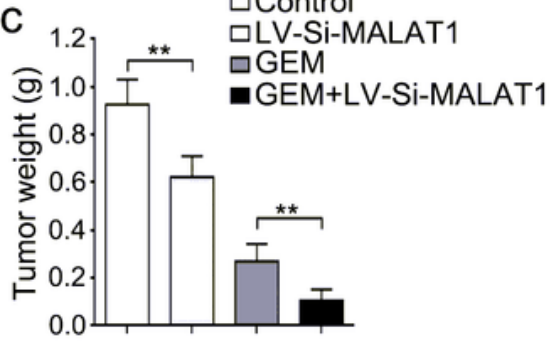

GEM

GEM+LV-Si-MALAT1
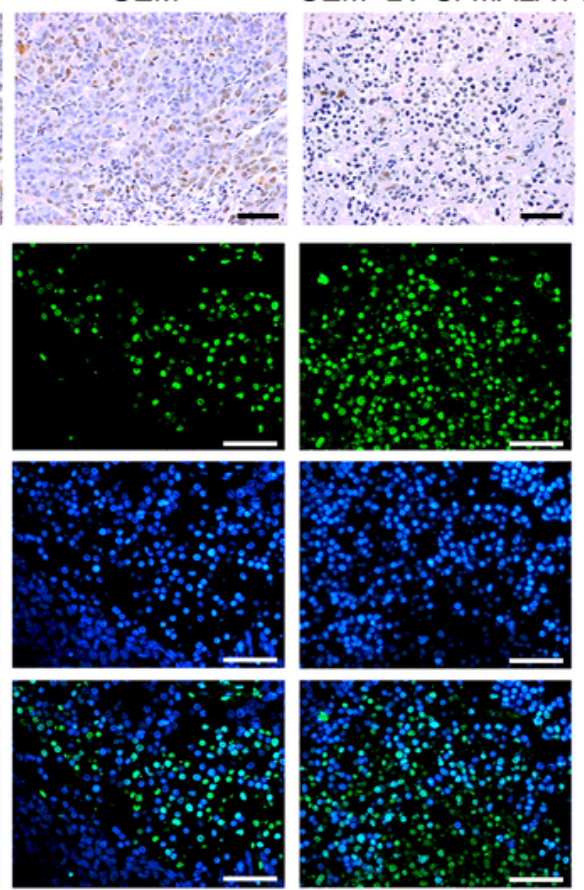

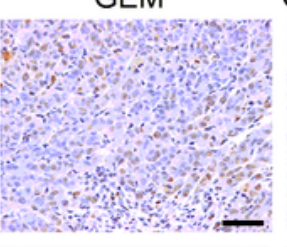

Figure 5

MALAT1 inhibition enhances the killing effect of gemcitabine in vivo. After transfection with LV-SiMALAT1, SW1990 cells were harvested and injected into the right flanks of athymic mice. After approximately 5 days, the mice were randomly divided into control, LV-Si-MALAT1, GEM, and GEM+ LV-SiMALAT1 groups. Gemcitabine was intraperitoneally injected at a dose of $20 \mathrm{mg} / \mathrm{kg}$ every 3 days. a Representative photographs of mice and tumors after 35 days of gemcitabine treatment. b The growth 
curve was delineated according to the volume measured every 5 days. c The weight of tumor in each group was presented. $d$ The representative expression levels of Ki-67 and TUNEL in tumor tissue sections were measured by immunohistochemistry and immunofluorescence assays, respectively (scale bar, 50 $\mu \mathrm{m})$. The data shown in the graphs are from three independent experiments. ${ }^{*} \mathrm{P}<0.01$.

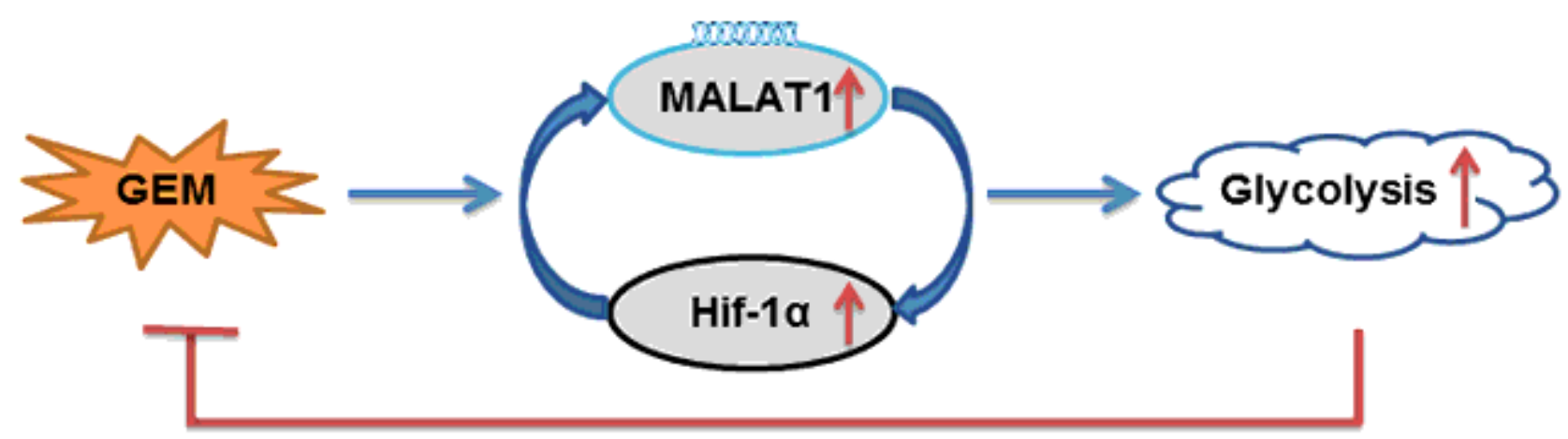

Figure 6

Schematic illustration demonstrating how the reciprocal loop of MALAT1 and HIF-1 a facilitates gemcitabine resistance in pancreatic cancer by enhancing glycolysis.

\section{Supplementary Files}

This is a list of supplementary files associated with this preprint. Click to download.

- Figs1.tif

- SupplementaryMaterial.docx 